\title{
Endoscopic Gastrojejunostomy for Superior Mesenteric Artery Syndrome Using Magnetic Compression Anastomosis
}

\author{
Hideaki Kawabata ${ }^{a}$,, Daiki Sone ${ }^{a}$, Katsutoshi Yamaguchia ${ }^{a}$ Naonori Inoue ${ }^{a}$, Yuji Okazaki ${ }^{a}$, \\ Yuki Ueda ${ }^{a}$, Misuzu Hitomi ${ }^{a}$, Masatoshi Miyata ${ }^{a}$, Shigehiro Motoi ${ }^{a}$
}

\begin{abstract}
An 89-year-old woman who was bedridden suffered repeated vomiting due to superior mesenteric artery syndrome (SMAS). We performed gastrojejunostomy via the magnetic compression anastomosis (MCA) technique because her situation was not improved by conservative therapy and because the operative risk was high. We prepared two neodymium magnets: a flat plate-shaped magnet $(15 \times 3 \mathrm{~mm})$ and a ring-shaped magnet of the same size. The ringshaped magnet which passed through a guidewire was pushed to the duodenum by an endoscope over the guidewire. The duodenal stricture was balloon-dilated in front of the magnet, and the magnet was pushed all together beyond the stricture and placed at the duodenojejunal junction. Subsequently, the flat plate-shaped magnet was delivered endoscopically to the stomach using a biopsy forceps. The magnets were attracted towards each other transmurally after one more flat plate-shaped magnet was added to the gastricside magnet. Completion of gastrojejunostomy was confirmed while retrieving the magnets 10 days after starting compression. She has been asymptomatic for 1 month since anastomosis. Endoscopic gastrojejunostomy using MCA was an effective, low-invasive treatment for SMAS.
\end{abstract}

Keywords: Magnetic compression anastomosis; Superior mesenteric artery syndrome; Gastrojejunostomy; Endoscopy

\section{Introduction}

Superior mesenteric artery (SMA) syndrome is a rare cause of proximal small bowel obstruction, the incidence of which is $0.1-0.3 \%$. It is caused by a narrow angle between the aorta and the SMA, resulting in the compression of the third portion of the duodenum [1]. Conservative management for SMA

Manuscript submitted September 25, 2019, accepted October 7, 2019

aDepartment of Gastroenterology, Kyoto Okamoto Memorial Hospital, Kyoto 613-0034, Japan

${ }^{b}$ Corresponding Author: Hideaki Kawabata, Kyoto Okamoto Memorial Hospital, 100 Nishinokuchi, Sayama, Kumiyama-cho, Kuze-gun, Kyoto 613-0034, Japan. Email: hkawabata@okamoto-hp.or.jp

doi: https://doi.org/10.14740/gr1229 syndrome, including fluid resuscitation, bowel rest, total parenteral nutrition and enteric feeding with a nasojejunal tube inserted past the obstruction, is initially attempted [2]. However, it often fails, and then surgical treatment such as laparoscopic duodenojejunostomy is needed to relieve the symptom $[1,3]$.

Magnetic compression anastomosis (MCA) was developed as a low-invasive, alternative treatment for enteric or biliary obstruction [4-8], which has been shown to consistently create histologically well-formed anastomoses with strength equal to or greater than that of hand-sewn or stapled anastomoses in animal studies [9-12]. Thus, favorable, clinical results in surgically assisted, minimally invasive gastro-enteric or entero-enteric MCA have been reported [6, 13]. However, whether the technique should be applied in the clinical setting is controversial due to the potential for severe postoperative complications, including anastomotic leakage and damage to unintended tissue between the mated magnets, which has been reported in several cases [4]. Furthermore, in several cases, prophylactic stent placement against post-anastomotic stricture induced stent-related complications, such as perforation, stent migration, and stent obstruction [5, 14].

We herein report the first case of endoscopic gastrojejunostomy using MCA for the treatment of SMA syndrome.

\section{Case Report}

An 89-year-old woman who was admitted to hospital and bedridden due to traumatic acute subdural hematoma was referred to our department with a 3-day history of repeated vomiting since her admission in June 2019. She had no history of significant surgical complications. Examination revealed a body mass index of $17.7 \mathrm{~kg} / \mathrm{m}^{2}$ and abdominal distension without tenderness. Abdominal computed tomography (CT) revealed a severely dilated stomach and significant dilatation of the duodenum up to the level of the distal third portion, which was sandwiched between the aorta and SMA (Fig. 1). The angle between the aorta and SMA was $14^{\circ}$, and the aorta-SMA distance was $5 \mathrm{~mm}$, which met the diagnostic imaging criteria for adult SMA syndrome [15]. Upper gastrointestinal endoscopy after nasogastric drainage showed narrowing of the third portion of the duodenum without mucosal abnormality; the endoscope was not able to pass through this site. Fluoroscopy showed a smooth, abrupt obstruction at the third portion of the duodenum. We performed gastrojejunostomy via the MCA 


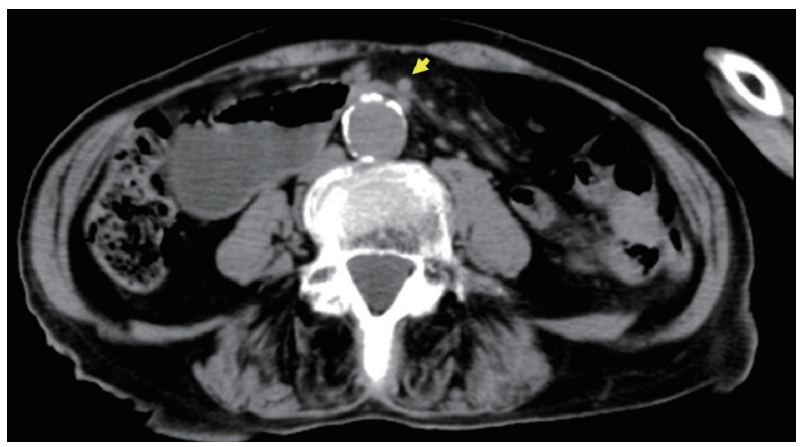

Figure 1. Abdominal CT revealed dilatation of the duodenum up to the level of the distal third portion, which was sandwiched between the aorta and SMA (arrow). CT: computed tomography.

technique because her situation did not improve after 1 month of conservative therapy, which included nil per os, nasogastric drainage and enteral nutrition via a W-ED tube (Japan Covidien Corporation, Tokyo), and because the operative risk was high. Written informed consent was obtained from the patient and her family.

We prepared two neodymium magnets: a flat plate-shaped magnet $(15 \times 3 \mathrm{~mm}$; Magfine, Sendai, Japan $)$ with a small hole of $3 \mathrm{~mm}$ in diameter and a ring-shaped magnet $(15 \times 3 \mathrm{~mm}$, internal diameter of $10 \mathrm{~mm}$ ). A nylon thread was passed through each of the holes. The placement of the magnets was planned based on fluoroscopy and CT. We then confirmed that there was no unintended tissue, such as small intestine or colon tissue adjacent to the anastomotic site at the predicted locations of the magnets. First, a guidewire (0.035 in; Hydra Jagwire, Boston Scientific, Japan) was inserted into the jejunum. Then, the ring-shaped magnet, which passed through the guidewire was pushed over the guidewire and delivered to the duodenum by a forward-viewing endoscope (GIF-Q260J; Olympus, Ja- pan). The duodenal stricture was dilated in front of the magnet by up to $16.5 \mathrm{~cm}$ using a dilating balloon (CRE Wire-guided, Boston Scientific, Japan) (Fig. 2a), and the magnet was pushed all together beyond the stricture by the endoscope and placed at the duodenojejunal junction. Subsequently, the flat plate-shaped magnet was delivered to the stomach endoscopically using a biopsy forceps. The magnets were attracted towards each other transmurally after one more flat plate-shaped magnet was added to the gastric-side magnet (Fig. 2b). We easily retrieved the magnets using a biopsy forceps 10 days after starting compression, and confirmed the completion of gastrojejunostomy (Fig. 3a, b). The patient then started direct swallowing training. She has been asymptomatic in 1 month since anastomosis although slight anastomotic stricture was confirmed endoscopically (Fig. 4).

\section{Discussion}

We showed that endoscopic gastrojejunostomy using MCA was useful as a minimally invasive treatment for SMAS syndrome. We found two clinical studies investigating the availability of endoscopic gastroenterostomy using MCA followed by the insertion of a self-expandable metal stent $[5,14]$. Chopita et al [5] demonstrated the feasibility, safety and efficacy of this technique for creating gastroenteric anastomosis: the procedure success rate was $88 \%$ ( 13 out of 15 patients), with four patients experiencing stent migration as a minor complication. Van Hooft et al [14] showed that the necessity of stenting led to serious morbidity, including stent migration, and even mortality due to stent perforation, while the endoscopic creation of gastroenteric anastomosis by magnetic compression was feasible and safe.

In the case of gastroenterostomy using MCA without stent insertion, Pichakron et al [10] revealed that only one of
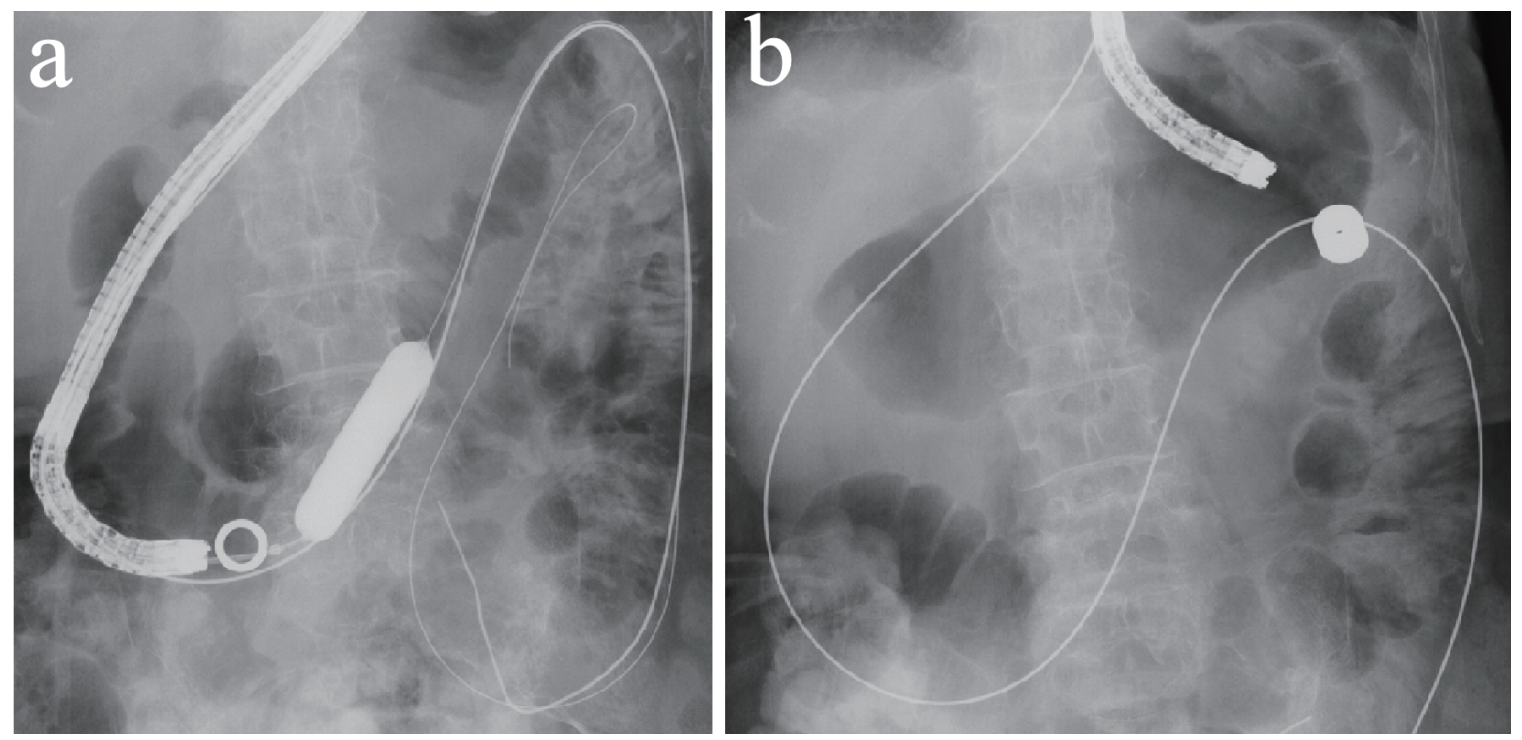

Figure 2. The duodenal stricture was dilated up to $16.5 \mathrm{~cm}$ using a dilating balloon in front of the magnet (a). The magnets were attracted towards each other transmurally between the stomach and duodenojejunal junction after one more flat plate-shaped magnet was added to the gastric-side magnet (b). 

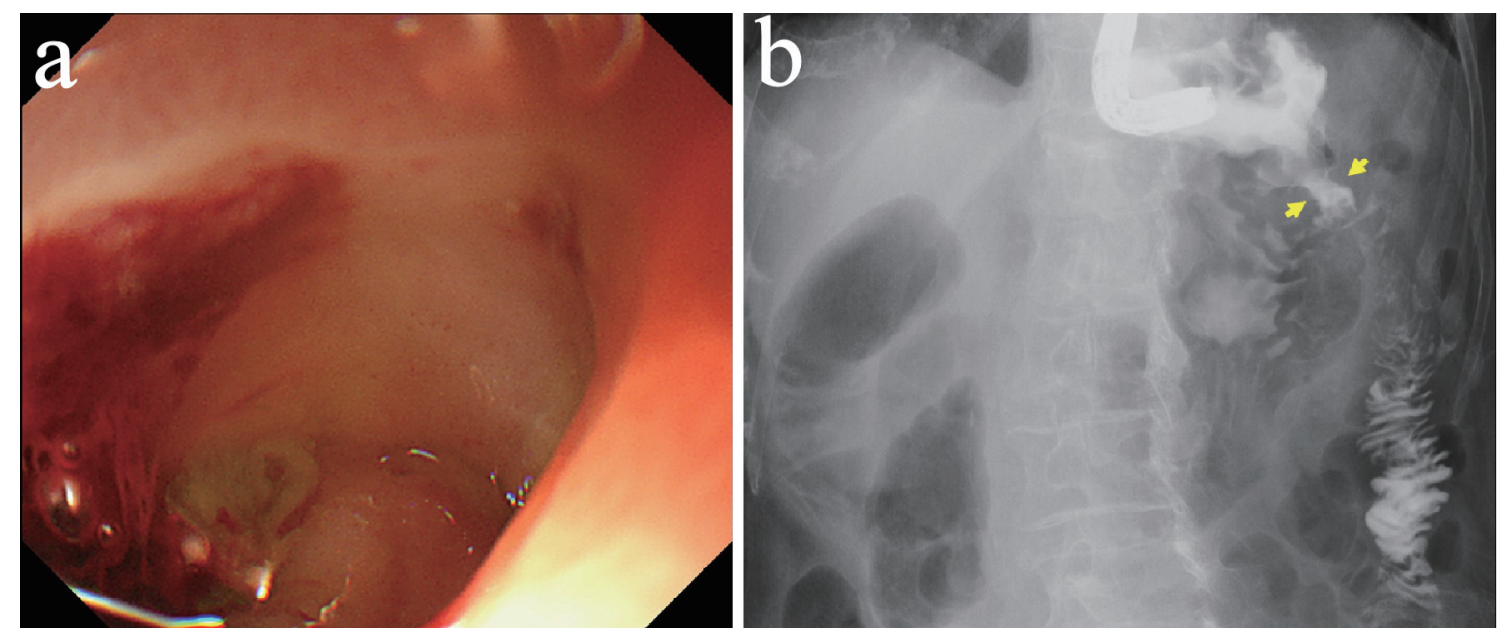

Figure 3. The completion of gastrojejunostomy was confirmed after retrieving the magnets (arrows) (a, b).

nine gastrojejunal anastomoses created by MCA showed stricture at 2 - 6 weeks after the procedure in an animal study. In contrast, Yamanouchi et al [4] reported that over $80 \%$ of gastroduodenal anastomoses created by MCA showed stricture, which necessitated repeated balloon dilation. In the present case, the patient has been asymptomatic; however, anastomotic stricture was detected 1 month after canalization. The continuous passage of digestive juices or foods can prevent severe anastomotic stricture. Considering the risk of severe complications due to stent placement, stent insertion should not be indicated after gastroenterostomy using MCA, and balloon dilation should be performed in cases of symptomatic anastomotic stricture.

In conclusion, endoscopic gastrojejunostomy using MCA was found to be an effective, low-invasive treatment for SMA

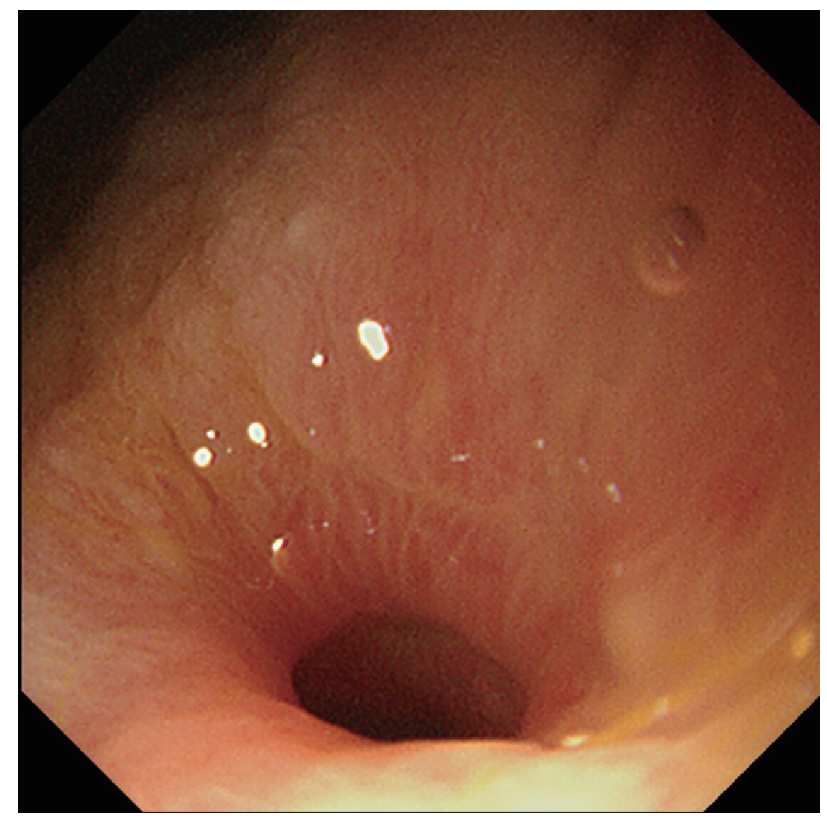

Figure 4. Slight anastomotic stricture was confirmed endoscopically 1 month after canalization. syndrome. Larger studies with longer follow-up periods are needed to confirm its utility and safety.

\section{Acknowledgments}

None to declare.

\section{Financial Disclosure}

None to declare.

\section{Conflict of Interest}

The authors declare that they have no conflict of interest.

\section{Informed Consent}

Written informed consent was obtained from the patient.

\section{Author Contributions}

H. Kawabata, D. Sone, K. Yamaguchi, N. Inoue, Y. Okazaki, Y. Ueda, M. Hitomi, and M. Miyata contributed to endoscopic diagnosis and treatment. M. Miyata and S. Motoi supervised the findings of this work. H. Kawabata wrote the manuscript with support from M. Hitomi and S. Motoi. All authors discussed the results and contributed to the final manuscript.

\section{References}

1. Van Horne N, Jackson JP. Superior mesenteric artery syndrome. In: StatPearls. Treasure Island (FL), 2019.

2. Merrett ND, Wilson RB, Cosman P, Biankin AV. Supe- 
rior mesenteric artery syndrome: diagnosis and treatment strategies. J Gastrointest Surg. 2009;13(2):287-292.

3. Chang J, Boules M, Rodriguez J, Walsh M, Rosenthal R, Kroh M. Laparoscopic duodenojejunostomy for superior mesenteric artery syndrome: intermediate followup results and a review of the literature. Surg Endosc. 2017;31(3):1180-1185.

4. Yamanouchi E, Kumano R, Kobayashi K, Hattori T, Matsumoto J, Oonishi T, Hayakawa M, et al. [Treatment for bowel or biliary obstruction by magnetic compression anastomosis development of Yamanouchi's method and its clinical evaluation]. J Nippon Med Sch. 2002;69(5):471475.

5. Chopita N, Vaillaverde A, Cope C, Bernedo A, Martinez H, Landoni N, Jmelnitzky A, et al. Endoscopic gastroenteric anastomosis using magnets. Endoscopy. 2005;37(4):313-317.

6. Graves CE, Co C, Hsi RS, Kwiat D, Imamura-Ching J, Harrison MR, Stoller ML. Magnetic compression anastomosis (Magnamosis): first-in-human trial. J Am Coll Surg. 2017;225(5):676-681 e671.

7. Jang SI, Choi J, Lee DK. Magnetic compression anastomosis for treatment of benign biliary stricture. Dig Endosc. 2015;27(2):239-249.

8. Kawabata H, Hitomi M, Inoue N, Kawakatsu Y, Okazaki Y, Miyata M. Intraductal ultrasonography as a local assessment before magnetic compression anastomosis for obstructed choledochojejunostomy. Gastroenterology Res. 2017;10(4):255-258.

9. Fan C, Ma J, Zhang HK, Gao R, Li JH, Yu L, Wu Z, et al. Sutureless intestinal anastomosis with a novel device of magnetic compression anastomosis. Chin Med Sci J. 2011;26(3):182-189.

10. Pichakron KO, Jelin EB, Hirose S, Curran PF, Jamshidi R, Stephenson JT, Fechter R, et al. Magnamosis II: Magnetic compression anastomosis for minimally invasive gastrojejunostomy and jejunojejunostomy. J Am Coll Surg. 2011;212(1):42-49.

11. Jamshidi R, Stephenson JT, Clay JG, Pichakron KO, Harrison MR. Magnamosis: magnetic compression anastomosis with comparison to suture and staple techniques. J Pediatr Surg. 2009;44(1):222-228.

12. Zhang H, Tan K, Fan C, Du J, Li J, Yang T, Lv Y, et al. Magnetic compression anastomosis for enteroenterostomy under peritonitis conditions in dogs. J Surg Res. 2017;208:60-67.

13. Toselli L, Martinez-Ferro M, Cervio G, Kwiat D, Imamura-Ching J, Graves CE, Gaston B, et al. Magnetic compression anastomosis (Magnamosis) for functional undiversion of ileostomy in pediatric patients. J Laparoendosc Adv Surg Tech A. 2017;27(12):1314-1317.

14. van Hooft JE, Vleggaar FP, Le Moine O, Bizzotto A, Voermans RP, Costamagna G, Deviere J, et al. Endoscopic magnetic gastroenteric anastomosis for palliation of malignant gastric outlet obstruction: a prospective multicenter study. Gastrointest Endosc. 2010;72(3):530-535.

15. Unal B, Aktas A, Kemal G, Bilgili Y, Guliter S, Daphan C, Aydinuraz K. Superior mesenteric artery syndrome: CT and ultrasonography findings. Diagn Interv Radiol. 2005;11(2):90-95. 